\title{
Estandarización contable en Colombia: avances y retos en la formación profesional contable
}

\author{
Zulma G. Sierra R. \\ Contadora Pública y Especialista en Auditoría de Sistemas, \\ Universidad Santo Tomás \\ Diego Fernando Católico Segura \\ Contador Público, Universidad Nacional de Colombia \\ Docente - Investigador Facultad de Contaduría Pública \\ Universidad Santo Tomás
}

\section{Resumen}

El presente artículo analiza el estado actual y cambios en la formación profesional contable. Para ello, se realiza una breve descripción de los avances en la estandarización contable en Colombia y se evalúa, de forma detallada, la percepción de estudiantes de cinco programas académicos de la ciudad de Bogotá, frente a aspectos que caracterizan la formación contable, bajo las condiciones actuales y futuras de la disciplina y la profesión.

\section{Palabras clave}

Normas Internacionales de Contabilidad (NIC); Normas Internacionales de Información Financiera (NIIF); Formación profesional contable; Plan de estudios; Convergencia; Armonización contable. 


\section{Introducción}

La internacionalización de los mercados ha implicado cambios en los factores económicos, políticos y culturales que caracterizan a los países que participan en el proceso. Factores como el conocimiento y la formación profesional cobran gran importancia en dicha dinámica, ya que determinan el valor agregado en las relaciones de producción.

La formación profesional contable no es ajena a este planteamiento. Es allí donde, surge una pregunta fundamental: ¿cuál debe ser el perfil y el conocimiento que debe caracterizar a los profesionales en Colombia?.

En este sentido, se han generado diversas opiniones sobre el tema. Un ejemplo de ello es lo enunciado por Mantilla (2002, p.158):

“... las condiciones del escenario internacional y global han hecho que surjan, en la práctica, dos tipos completamente diferentes de contadores, según se trate de si participan en mercados de capitales (contaduría y tecnología de la información, esto es, una contaduría caracterizada por la integración técnica), o según se trate de si participan en mercados locales y regionales (contaduría para PYME, esto es, una contaduría caracterizada por aportar soluciones integrales)... El asunto ha ido todavía más lejos y ha conllevado una nueva transformación paradigmática: la superación de la contaduría pública mediante la contaduría profesional".

Propuestas de revisión sobre la formación de los estudiantes de contaduría pública y la permanente actualización y desarrollo de estudios posgraduales por parte de los contadores públicos titulados, se han vuelto imperativos en este proceso de globalización. 
No obstante y aunque el proceso de estandarización contable en Colombia no se ha consolidado, las universidades trabajan en el diseño de programas académicos, de pregrado y posgrado, que respondan a las nuevas exigencias del contexto nacional e internacional.

Es así, que en el presente artículo se analiza el estado actual y cambios en la formación profesional contable. Para ello, se abordan los avances en la estandarización contable en Colombia y se evalúa la percepción de estudiantes de cinco programas académicos de la ciudad de Bogotá, frente a aspectos que caracterizan la formación contable, bajo las condiciones actuales y futuras de la disciplina y profesión.

\section{Avances de la estandarización contable en Colombia}

En los últimos años en Colombia, se ha originado una serie de propuestas por parte del Estado para la aplicación de estándares internacionales de contabilidad, las cuales no han logrado la aceptación general y, por el contrario, han encontrado obstáculos para su consolidación.

Ejemplo de lo anterior, son dos propuestas que se presentaron en el año 2003 y 2006. La primera de ellas, el 10 de diciembre de 2003, en la cual el Gobierno Colombiano en cabeza de un comité técnico interinstitucional ${ }^{1}$ presentó el borrador de proyecto de ley de intervención económica: "por medio del cual se señalan los mecanismos por los cuales se adoptan en Colombia los estándares internacionales de contabilidad, auditoria y contaduría; se modifican el Código de

\footnotetext{
${ }^{1}$ Conformado por los siguientes entes: Ministerio de Hacienda y Crédito Público, Ministerio de Comercio, Industria y Turismo, Superintendencia de Valores, Superintendencia Bancaria, Superintendencia de Sociedades, Contaduría General de la Nación, Dirección de Impuestos y Aduanas Nacionales, Departamento Nacional de Planeación
} 
Comercio, la normatividad contable, y se dictan otras disposiciones relacionadas con la materia".

La segunda, por parte de la Superintendencia de Sociedades al promover un borrador de proyecto de decreto que deroga el decreto 2650 de 1993 y elimina los ajustes integrales por inflación, con el sustento de ser medidas necesarias para poder acercar a Colombia a los procesos de convergencia contable ${ }^{2}$.

Debe reconocerse que, dada la tradición legalista del país, estos esfuerzos buscan concretar lo señalado por el artículo 63 de la ley 550 de 1999:

"ART.63. Armonización de las normas contables con los usos y reglas internacionales. Para efectos de garantizar la calidad, suficiencia $y$ oportunidad de la información que se suministre a los asociados y a terceros, el Gobierno Nacional revisara las normas actuales en materia de contabilidad, auditoria, Revisoría fiscal y divulgación de información, con el objeto de ajustarlas a los parámetros internacionales y proponer al Congreso las modificaciones pertinentes".

Lo anterior supone, adoptar un cuerpo normativo en materia contable que llegaría a modificar o eliminar la normatividad Colombiana actual, tanto en sus componentes básicos (normas básicas) como en su componente técnico y metodológico (normas técnicas).

\footnotetext{
${ }^{2}$ Proyecto de Borrador de Decreto. Por medio del cual se deroga el Plan Único de Cuentas y dicta otras disposiciones en materia contable. Superintendencia de Sociedades. Abril de 2006.
} 
Aunque estas propuestas no han sido consolidadas, el problema real existente, no es tanto de corte normativo sino económico ${ }^{3}$, es decir, más allá de lo expuesto en normas que se deben cumplir, es lo que realmente el mercado, la disciplina y la misma profesión exigen y deben enfrentar.

Reconocer las tendencias del mercado y los procesos de integración económica que Colombia decidió abordar, implica el desarrollo o captura de nuevos conocimientos y habilidades en distintas áreas, para aquellos agentes que intervienen en esta dinámica.

En el caso particular de la contabilidad y la contaduría, los cambios antes expuestos, no solo implican modificaciones del orden normativo técnico, sino que impactan la formación, el ejercicio y las prácticas contables colombianas, ya que temas como, por ejemplo, el reconocimiento, la revelación y la valoración se hacen críticos en este contexto, por lo cual se requiere de profesionales competentes con dominio en temas contables (marcos conceptuales, reguladores, normas técnicas, entre otras) que suponen operaciones en contextos internacionales, el uso de tecnologías de la información y el manejo de una segunda lengua.

En este orden de ideas, se requiere que los profesionales, estén formados integra e interdisciplinariamente para generar habilidades (intelectuales, interpersonales, y de comunicación); valores profesionales (integridad, objetividad, independencia, ética, sensibilidad social y compromiso de aprendizaje), y conocimiento (que en lo referente a contabilidad, integra la general, la especializada y la internacional) (Cardona, 2004, p.155).

\footnotetext{
${ }^{3}$ Colombia actualmente esta en un proceso de apertura económica con la firma y la participación activa de acuerdos comerciales con la Organización Mundial del Comercio, OECD, Comunidad Andina de Naciones (CAN), entre otros, interactuando productos, bienes y servicios; lo mismo esta sucediendo con el conocimiento, debido a que necesariamente el permitir la entrada de bienes tangibles genera el garantizar un mínimo de información contable y/o financiera que se maneje con las entidades del exterior.
} 
Lo anterior tiene relación con el método de enseñanza que actualmente sustenta la formación de los estudiantes en las Instituciones de Educación Superior. Es así, que más allá de la transmisión de un conjunto de conocimientos, se debe orientar al estudiante en el abordaje de las problemáticas actuales de la disciplina y la profesión. Es así, que surgen dos interrogantes: ¿Cuál es el papel que juegan las Universidades en las cuales se forman los profesionales contables? y ¿Cuál es la percepción que tienen los estudiantes sobre la formación que están recibiendo?.

\section{El papel de las universidades}

La educación superior se enfrenta a retos sin precedentes bajo el impacto de la globalización, el crecimiento económico basado en el conocimiento y la revolución de la comunicación y la información. Estos retos pueden ser percibidos como amenazas u oportunidades, para los cuales hay que estar preparados y anticiparse a los hechos, con la apropiación de un marco regulador efectivo y eficiente, con programas de calidad, innovadores y flexibles, es decir, con programas de mejoramiento continuo, completos, útiles, actualizados y de fácil acceso o consulta a los estudiantes, con alto sentido de pertenencia que dejen constancia de la responsabilidad social que le compete a la universidad, sin importar el escenario donde ésta se desenvuelva.

Debido a lo anterior, los programas académicos juegan un papel de suma importancia en el desarrollo de esta profesión. Se busca que los planes de estudios sean acordes a los diferentes procesos, sucesos y actividades a la que diariamente se ven enfrentados los profesionales contables. 
Actualmente las universidades tiene el pensum que se va adecuando a las necesidades que el mercado y el entorno va solicitando. Sin embargo, el entorno nacional ha demostrado que la evolución no ha sido tan rápida en relación a las necesidades que tienen el mercado colombiano y del exterior. Igualmente, surge la necesidad de contemplar en su plan de estudios el abordaje de los elementos que caracterizan a los reguladores (nacionales e internacionales), los marcos conceptuales, cuerpos normativos y demás elementos de los sistemas contables nacional e internacional.

Un ejemplo de esto es el abordaje de aplicaciones de Normas Internacionales de Contabilidad y su comparación con la Normatividad Colombiana, identificando diferencias conceptuales, técnicas y metodológicas.

Debido a que el tema ha ocupado un gran índice de preocupación, surge el interés particular por analizar la situación actual en la formación profesional contable, a través de la indagación de lo que piensan los futuros profesionales frente a puntos críticos que caracterizan el estado actual y futuro de la estandarización contable en Colombia.

Según los programas curriculares de las Universidades Santo Tomás, Javeriana, Central, Nacional y Externado, el tema de las normas internacionales de contabilidad aplicadas en Colombia es de continua investigación y estudio. Es así, que se han originado diferentes formas para abordar dicha problemática, como son los grupos de estudio, conferencias y convenios. En este último aspecto, estas universidades han venido consolidando un convenio para desarrollar, mejorar, 
complementar, interactuar conocimiento, estudiantes y problemáticas de estudio acerca del futuro contable en Colombia desde su enseñanza ${ }^{4}$.

En este sentido, existe evidencia que las Universidades vienen trabajando en la consolidación de sus programas académicos a las nuevas tendencias de la disciplina y profesión. Sin embargo, lo anterior no quiere decir que ya este todo terminado, sino que al contrario se ha iniciado un proceso que se debe continuar.

\section{Percepción de los estudiantes}

Como se expuso anteriormente, no solo es necesario reconocer el esfuerzo adelantado por las Universidades, sino que también, es importante evidenciar cómo los estudiantes perciben dichos cambios a través de lo que experimentan en su vivir académico.

En este sentido, se adelantó un sondeo en el 2004, en estas mismas instituciones, para determinar, la percepción que tienen los estudiantes sobre el tema en cuestión y cómo ha sido su formación sobre el mismo ${ }^{5}$.

Este sondeo determinó tres aspectos que abordan el impacto en la realidad de adoptar las normas internacionales de contabilidad, lo cual se soportó en una

\footnotetext{
${ }^{4}$ Convenio Marco de Cooperación Académica para los Programas de Contaduría Publica, el cual fue suscrito entre varias universidades entre ellas (Pontificia Universidad Javeriana, Universidad Central, Universidad Externado de Colombia, Universidad Nacional de Colombia y la Universidad Santo Tomas), el cual cumple con un objetivo de desarrollar relaciones académicas y científicas entre las Universidades en sus programas de Contaduría Pública. A este convenio se han sumado las Universidades de Antioquia, Valle, EAFIT, Autónoma de Bucaramanga y Javeriana de Cali.

${ }^{5}$ Este sondeo fue realizado en el mes de agosto de 2004 a una población total de 288 estudiantes de últimos semestres (9-10-11). Los estudiantes encuestados pertenecen a las Universidades Javeriana (41 estudiantes), Externado (34 estudiantes), Nacional (23 estudiantes), Central (90 estudiantes) y Santo Tomas (100 estudiantes). Aunque dicho sondeo se realizó en el 2004, los resultados expuestos guardan vigencia, soportándose en los avances realizados y en la percepción que aún tienen los estudiantes sobre el tema.
} 
encuesta constituida por 10 preguntas, algunas cerradas de selección múltipla con una única respuesta y algunas con múltiple respuesta; así mismo, se desarrollaron preguntas que sus respuestas supone una respuesta con posibilidad en ampliar la misma.

Los tres aspectos en cuestión son:

1). Efectos en la contabilidad financiera y tributaria,

2). Regulación y,

3). Formación profesional.

Para dar respuesta al primer aspecto, efectos en la contabilidad financiera y tributaria, se diseñaron y aplicaron las siguientes preguntas:

- ¿Cuáles cree usted que son los efectos tributarios de aplicar las NIC en Colombia?.

- ¿La adaptación de estándares normativos en materia contable, implica un cambio en el sistema tributario Colombiano?.

- ¿Para asumir la estandarización contable, que posición debe asumir el Gobierno de Colombia?

Las anteriores preguntas ambientan sobre la opinión que tienen los estudiantes acerca de los efectos en la contabilidad financiera y tributaria dada la internacionalización contable y teniendo en cuenta que el contador debe saber como mínino el marco legal y conceptual del proceso que debe afrontar, así como, los cambios y los efectos en los aspectos tributarios. 
Frente a lo anterior, se propone el analizar temas como eliminar la dependencia de lo contable en lo fiscal, así como otros aspectos que evalúan el contexto actual de la estandarización en Colombia.

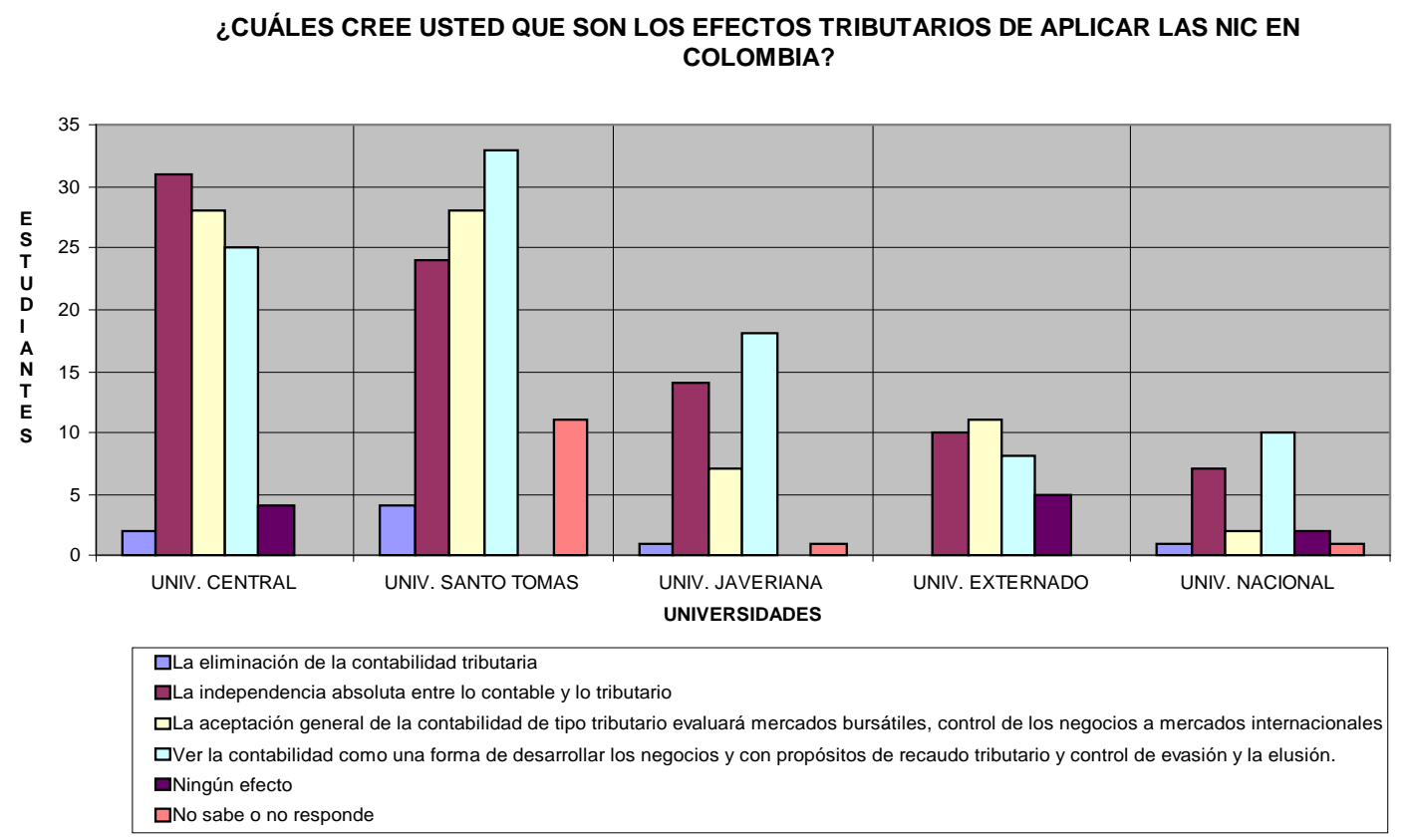

Fuente: Elaboración propia.

Después de indagar si se considera que la estandarización contable en Colombia implica un cambio en el sistema tributario, se encuentra que el $33 \%$ de los estudiantes creen que el efecto directo que traería la estandarización en este aspecto, es ver a la contabilidad como una forma de desarrollar los negocios y no con propósitos de recaudo tributario, control de evasión y elusión fiscal. Así mismo, se evidencia que un $30 \%$ de los encuestados ven como efecto la independencia absoluta entre lo contable y lo tributario. 
LA ADAPTACIÓN DE ESTANDARES NORMATIVOS EN MATERIA CONTABLE, ¿IMPLICA UN CAMBIO EN EL SISTEMA TRIBUTARIO COLOMBIANO?

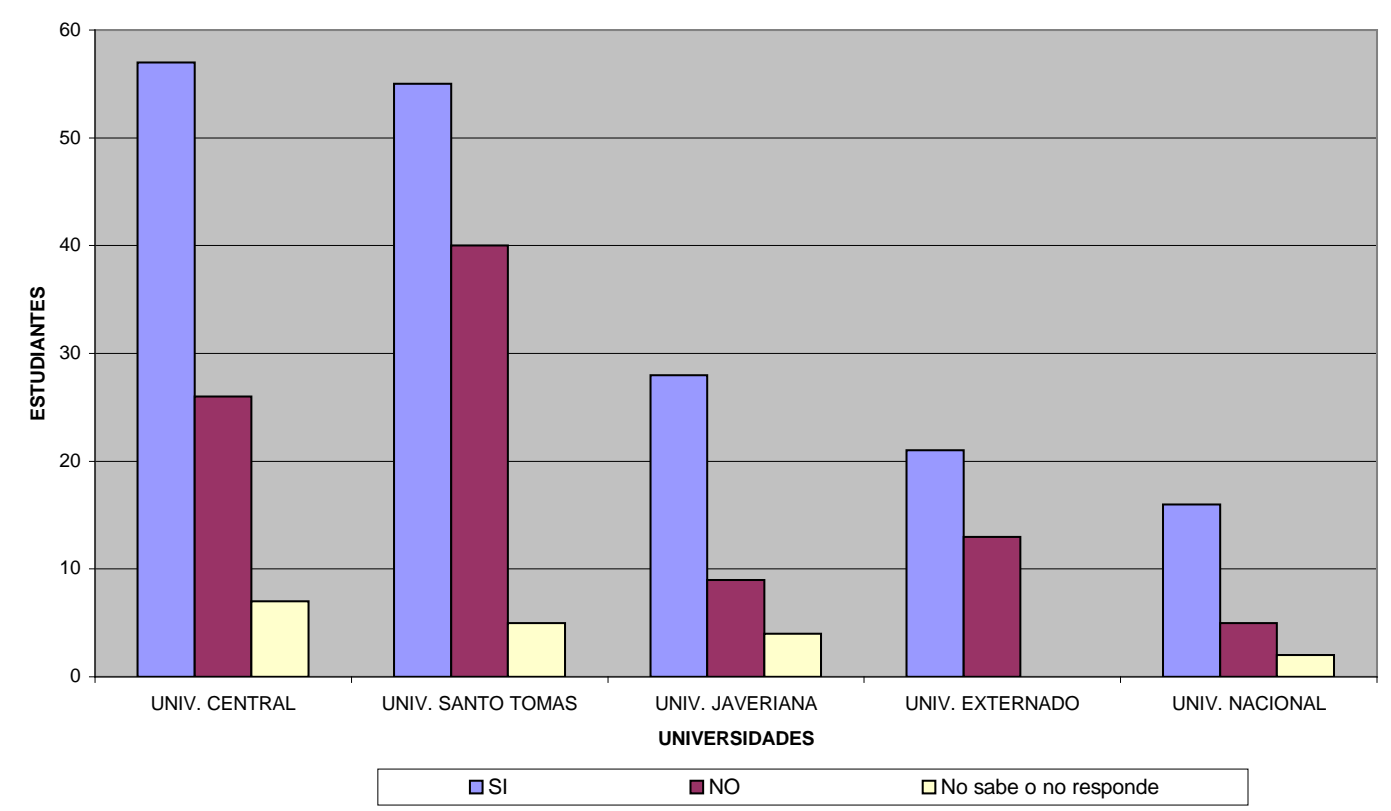

Fuente: Elaboración propia.

La adaptación de estándares normativos no solo en Colombia sino en cualquier país, puede o no tener un cambio en el sistema tributario. Los estudiantes afirmaron en un $61 \%$ que si implica un cambio en el sistema tributario Colombiano. Lo anterior, se fortalece con la idea que las normas internacionales fueron diseñadas en modelos donde la contabilidad es independiente de lo tributario, en entornos diferentes al que actualmente tiene Colombia. El 32\% afirma que no tiene implicación los estándares en materia contable en dicho aspecto. 


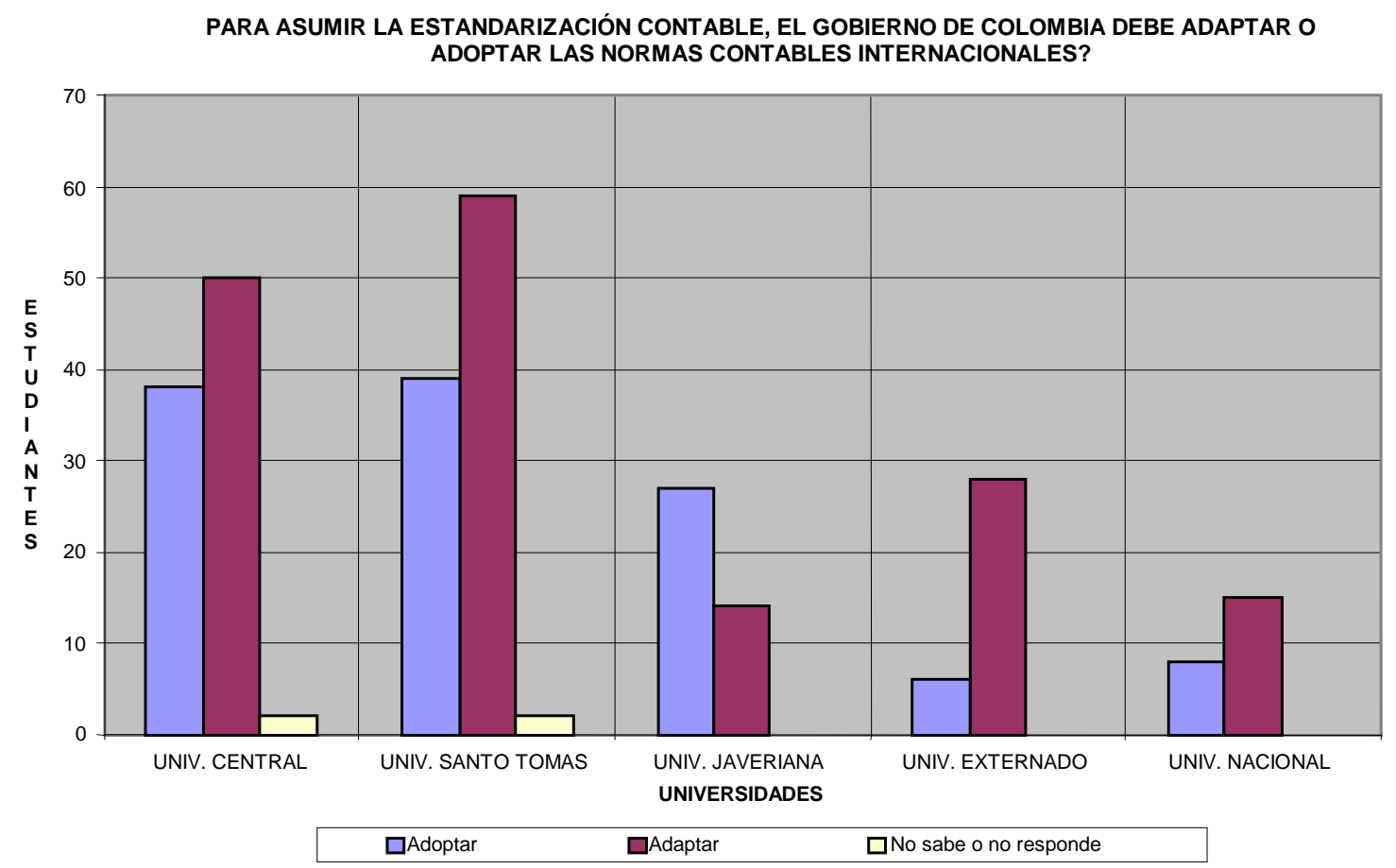

Fuente: Elaboración propia.

En algunas exposiciones de escritores como Mantilla, afirman que:

“adaptar no es corregir, mejorar o complementar el DR. 2649 de 1993 sino hay que derogarlo junto con sus normas reglamentarias y más bien adoptar las NIC-NIIF, para aquellas entidades que participen en mercados que coticen en bolsa" (Mantilla, 2001, p. 110).

La respuesta de lo que piensan los estudiantes arrojó como resultado que un 41\% piensa que es mejor adoptar las normas internacionales y el 58\% que se deberían adaptar.

Para seguir con el tema de la estandarización, el segundo aspecto a analizar es la regulación, para lo cual se diseñaron y aplicaron las siguientes preguntas: 
- ¿Creé Usted que los decretos 2649 y 2650 de 1993 y sus reglamentarios, ya cumplieron su ciclo?

- Según usted, ¿cuál sería la entidad competente, llegado el caso, para el reemplazo de la Junta Central de Contadores?

Teniendo en cuenta que el marco regulatorio en Colombia se caracteriza por estar conformado por un cuerpo normativo complejo y un gran número de instituciones regulatorias se hace necesario evaluar cuál será el impacto que puede tener el derogar algunos decretos y sustituir instituciones.

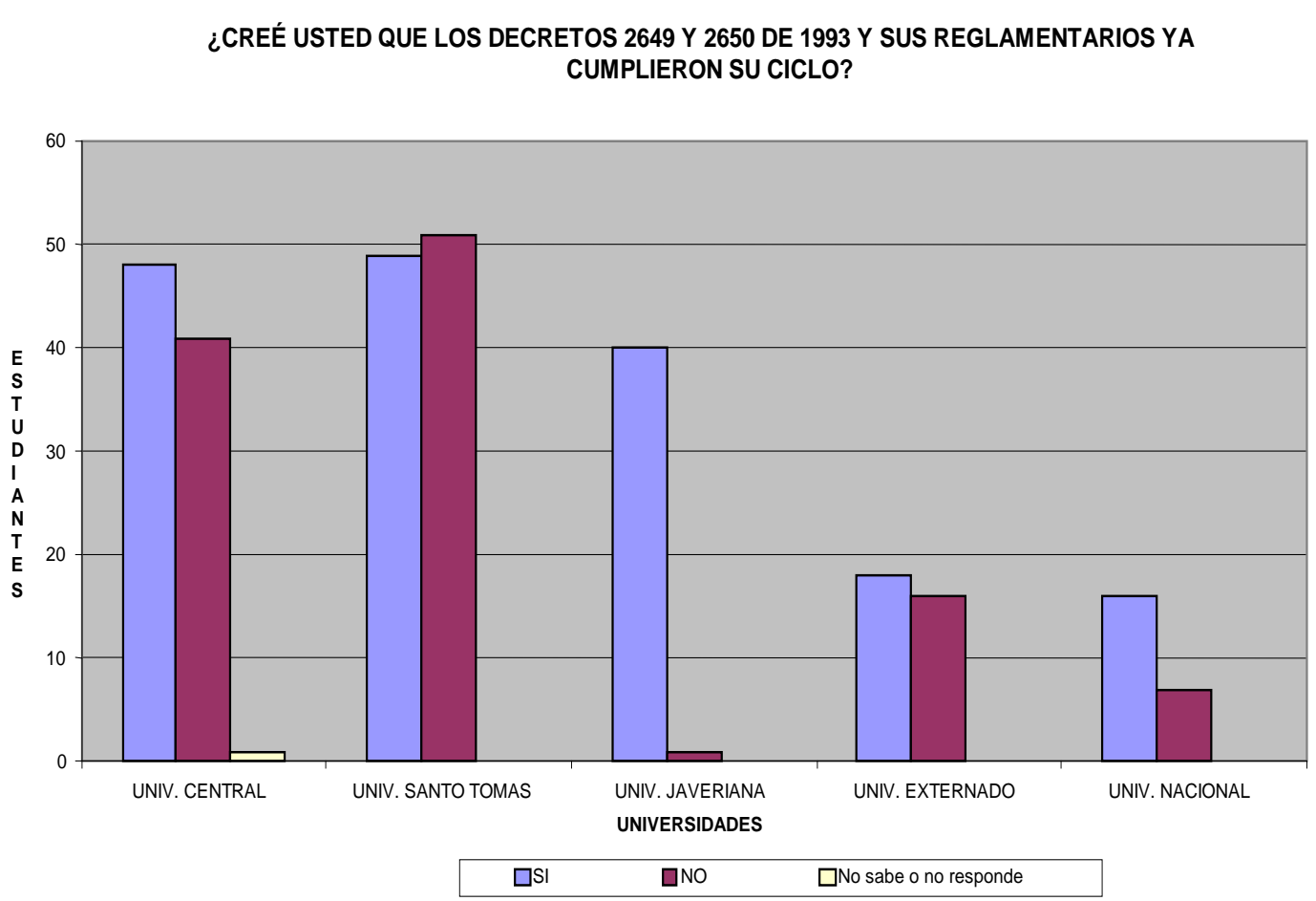

Fuente: Elaboración propia.

En Colombia los decretos reglamentarios 2649 y 2650 de 1993 son la base de la normativa contable del país; no obstante, hay que tener en cuenta que si se asume la estandarización contable, es decir la adopción o la adaptación de las NIC - NIIF en Colombia, vale la pena preguntarse si estos decretos ya cumplieron 
su ciclo o siguen siendo vigentes frente a las nuevas tendencias de globalización. Como respuesta a esta pregunta s encuentra que el 59\% están de acuerdo en que ya cumplieron su ciclo y deben ser actualizados a la necesidades del momento, sin dejar a un lado que se pueden rescatar algunos conceptos y teorías que se aplican en el ejercicio normal de la profesión por medio de estos decretos; no obstante, existe un porcentaje de $40 \%$ que admiten que aún no han cumplido su ciclo, ya que conservan en su esencia la base principal de la técnica contable.

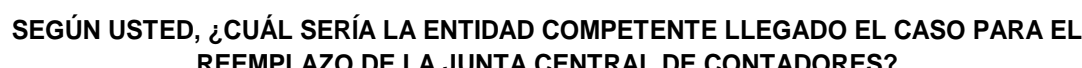
REEMPLAZO DE LA JUNTA CENTRAL DE CONTADORES?

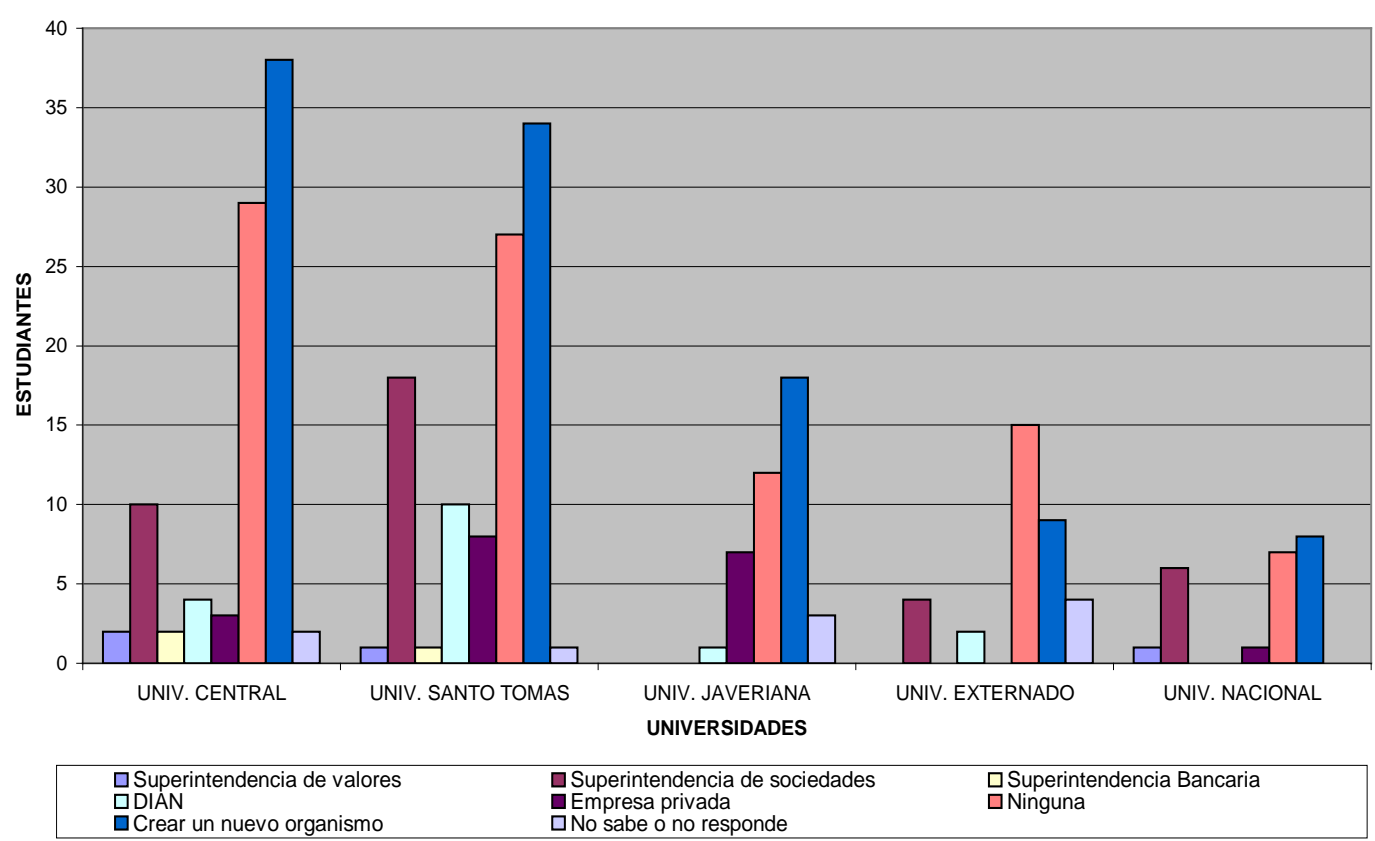

Fuente: Elaboración propia.

La Junta Central de Contadores (JCC) es el tribunal disciplinario para los contadores públicos en Colombia, el cual data desde el año 1956 hasta hoy y seguirá por tiempo indefinido hasta que se vea la necesidad de reemplazarla o reforzarla con otros elementos que ayuden a controlar el ejercicio profesional en el país. 
Por tal motivo, se escogieron 6 posibles entidades, que llegado el caso, serian el reemplazo de la Junta Central de Contadores. Los estudiantes frente a este tema tienen opiniones contrarias. El 37\% de los encuestados venla necesidad de crear u organismo nuevo y un $31 \%$ creen que ninguna entidad debería ser el reemplazo de la JCC. Esto lleva a pensar que en lugar de reemplazar la Junta, se debería reformar o reestructura para que sea este organismo el encargado de seguir controlando el ejercicio profesional contable.

Por último, tenemos la formación profesional como aspecto en análisis, para lo cual las preguntas aplicadas fueron:

- Con relación a la estandarización de la contabilidad a nivel mundial, el contador ¿qué es lo que debe saber?.

- ¿Conoce universidades que estén trabajando temas relacionados con la adopción o adaptación de las normas internacionales en Colombia?

- ¿Qué elementos de la estandarización considera usted que debe tener un programa de Contaduría Pública en Colombia para que el profesional egresado sea competitivo en el mundo actual?.

Como tema para cerrar el análisis de la internacionalización contable en Colombia, es necesario conocer cómo las instituciones formadoras de los futuros profesionales tienen una estructura académica para enfrentar este nuevo reto ${ }^{6}$.

6 Es preciso aclarar que en el proyecto de ley de intervención económica se evalúa las situaciones de la autonomía universitaria (articulo 22) y los cambios que debe asumir el profesional contable para enfrentar este nuevo reto (articulo 23 al 25). 


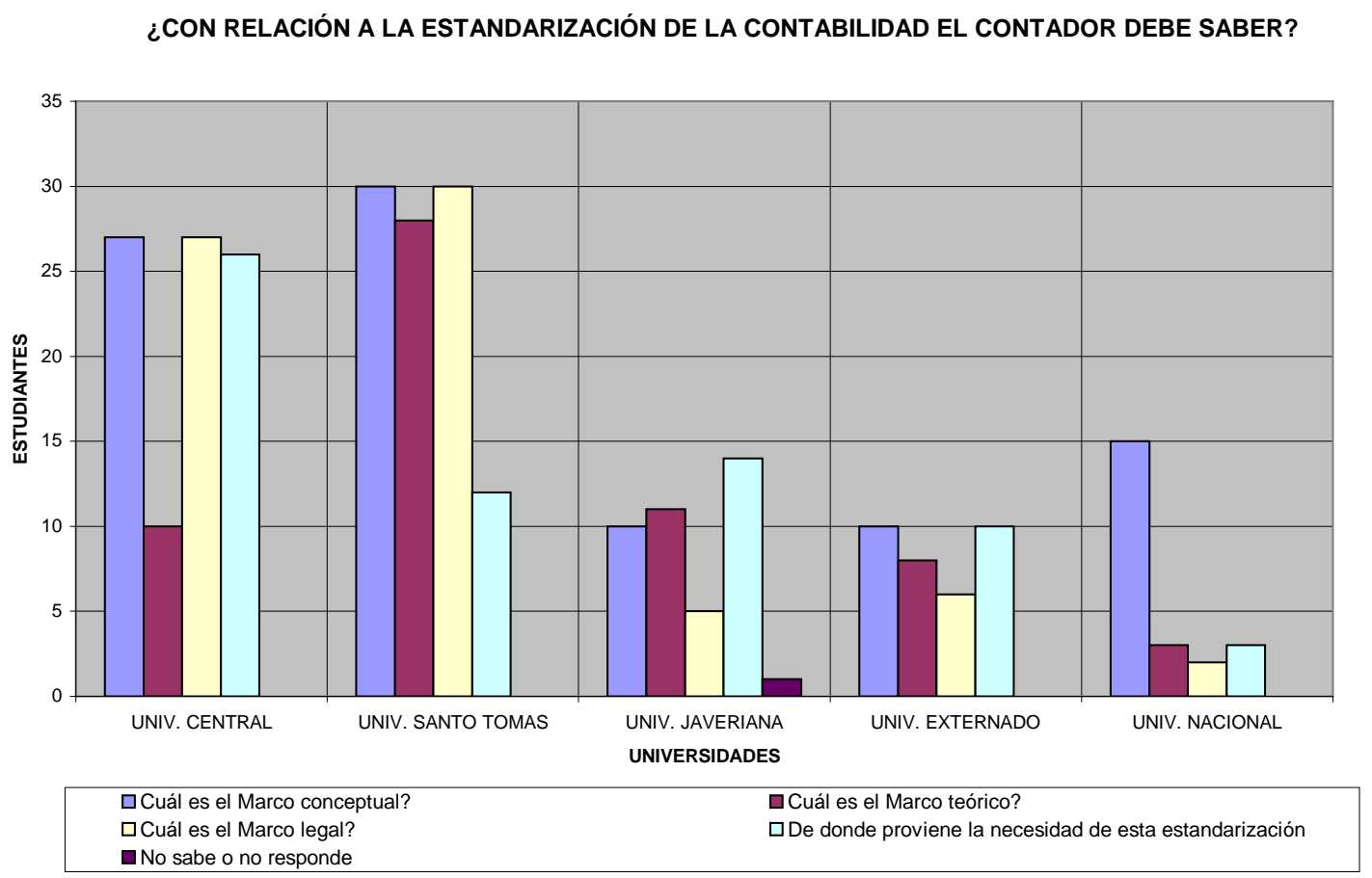

Fuente: Elaboración propia.

Como conclusión se determina que conocer el marco conceptual (32\%) y legal (24\%), son los aspectos que los estudiantes de las universidades consideran que debe manejar un profesional actualizado. 


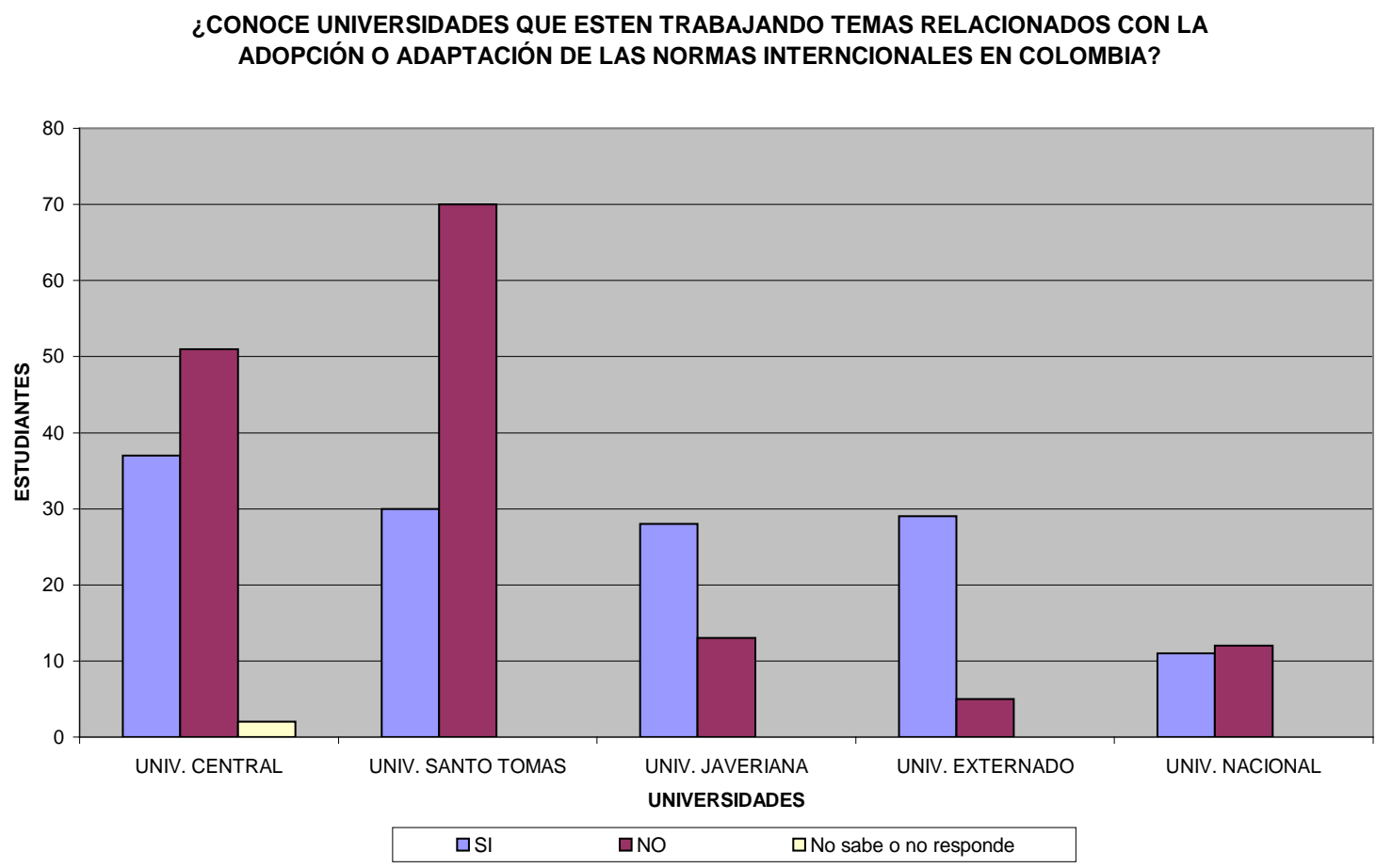

Fuente: Elaboración propia.

En esta pregunta el estudiante, además de indicar si conoce o no sobre universidades que estén trabajando el tema, debía indicar, en caso de una respuesta positiva, el nombre de la institución. El resultado obtenido de los estudiantes encuestados demostró que el 52\% no conoce universidades que estén trabajando temas relacionados con la adopción o adaptación de las normas internacionales en Colombia. La anterior respuesta tiene gran incidencia por el número de estudiantes de la Universidad Santo Tomás que respondieron negativamente ( $47 \%$ de esta repuesta). Sin embargo, el $47 \%$ de los estudiantes encuestados cree conocer que la Universidad Javeriana (81), Externado (37), Central (19) y Nacional (11), están trabajando temas relacionados con la adopción o adaptación de las normas internacionales en Colombia. 
PROGRAMA DE CONTADURÍA PÚBLICA EN COLOMBIA PARA QUE EL PROFESIONAL EGRESADO SEA COMPETITIVO?

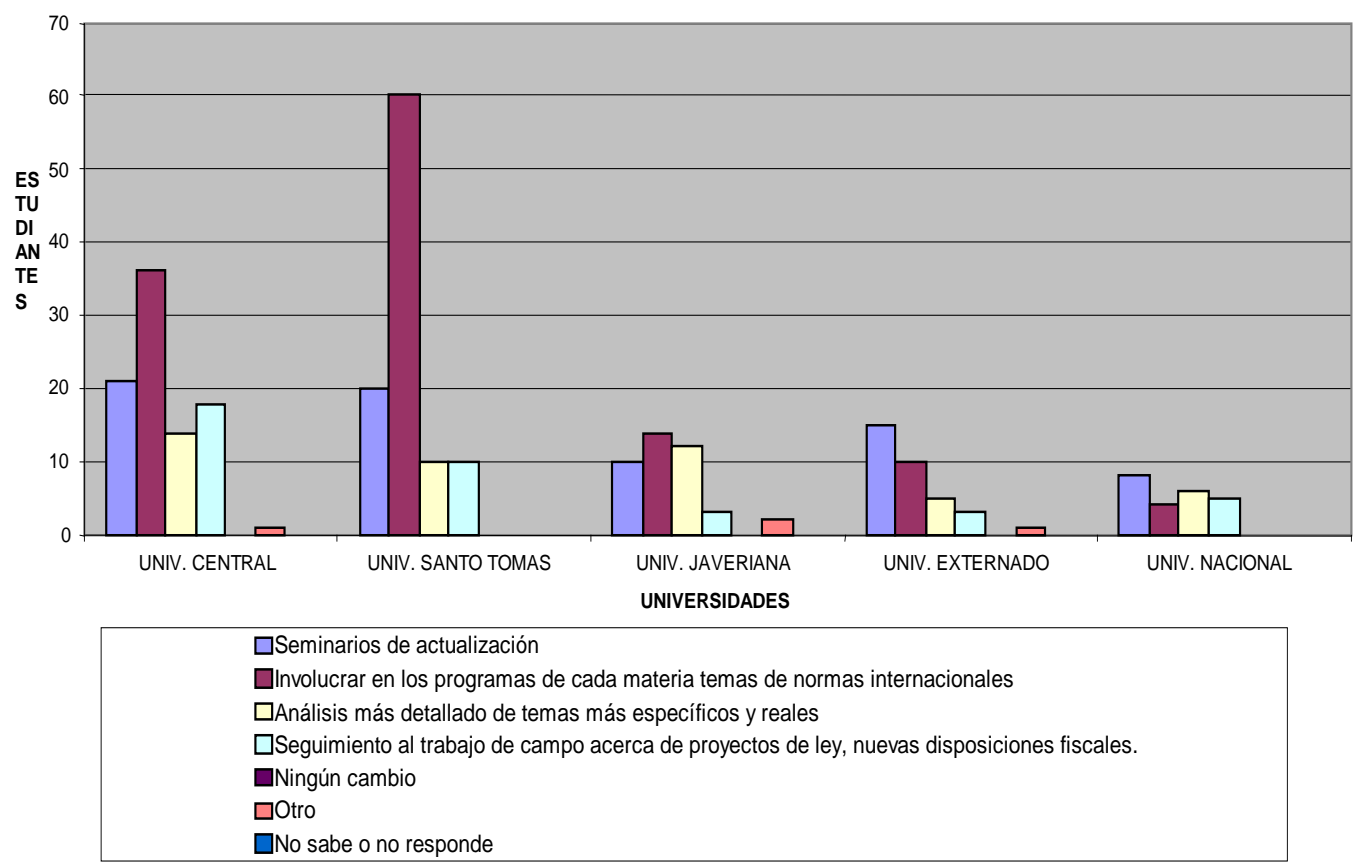

Fuente: Elaboración propia.

En esta pregunta la intención no es en ningún momento cuestionar los programas académicos que tenga cada centro educativo, sino permitir al estudiante mirar otras posibilidades, desde el momento que se está preparando académicamente, tener herramientas de conocimiento para poder afrontar un cambio en la estructura contable en el país. Como resultado de esta pregunta tenemos que el $43 \%$ considera que es importante involucrar en los programas de cada materia temas de normas internacionales y con un $26 \%$ tener seminarios de actualización.

\section{Conclusiones}

1. La adopción de estándares internacionales incluye cambios en la regulación, la aplicación y la formación del contable, entre otros. Este último, supone un 
papel activo de las universidades y estudiantes, modificando los programas académicos, en cuanto al plan de estudios, espacios académicos, la interdisciplinariedad y la forma como estos se desarrollan.

2. Los avances que se han realizado en las universidades para abordar el tema han sido importantes. Sin embargo, el camino por recorrer sigue siendo extenso, para lo cual se invita a los programas académicos de las distintas universidades a repensar las temáticas impartidas y el perfil del profesional contable que se está formando.

3. Según el sondeo realizado, la percepción que tienen los estudiantes de Contaduría Pública, frente a los avances y las temáticas que se vienen desarrollando en los programas académicos, así como en aspectos críticos en la estandarización contable en Colombia, es variada frente a cada una de las preguntas, pero con un número de semejanzas importantes frente a lo que se piensa.

4. Aspectos como la adopción de los estándares internacionales, los efectos en el sistema tributario, la separación de lo contable y fiscal, y la eliminación del decreto 2649 y 2650 de 1993, marcan los puntos de mayor interés entre los estudiantes y las respuestas con mayor coincidencia entre los resultados obtenidos en el ejercicio realizado.

5. El tema de la formación profesional contable es un tema cada vez más complejo y no fácil de abordar, dadas las nuevas condiciones del contexto y los cambios que se presentan en el mismo, es por eso, que pensar que el adoptar estándares internacionales y enseñar dicho cuerpo normativo sea la solución para el desarrollo de la disciplina y profesión contable en Colombia, puede ser un error, ya que esto no es el fin, sino que es un paso que 
representa un avance frente a temáticas internacionales, pero el cual siempre se estará frente a nuevas condiciones y desarrollos científicos, disciplínales y profesionales.

\section{Bibliografía}

Cardona Arteaga, John; Zapata Monsalve, Miguel Ángel (2004). Estándares Internacionales para la Formación del Contador Público. En: Revista Contaduría Universidad de Antioquia, No. 44, enero - junio, pp. 152-163.

Comité Interinstitucional (2003). Borrador Proyecto de ley de intervención económica. Por medio de la cual se señalan los mecanismos por los cuales se adoptan en Colombia los estándares internacionales de contabilidad, auditoría y contaduría, se modifican el Código de Comercio, la normatividad contable y se dictan otras disposiciones relacionadas con la materia.

Congreso de la República de Colombia (1999). Ley 550. "Por la cual se establece un régimen que promueva y facilite la reactivación empresarial y la reestructuración de los entes territoriales para asegurar la función social de las empresas y lograr el desarrollo armónico de las regiones y se dictan disposiciones para armonizar el régimen legal vigente con las normas de esta ley".

Eichengreen, Barry (2000). Hacia una nueva arquitectura financiera internacional. Mexico D.F.: Oxford University Press, pp. 1-40.

Espinosa, German (2002). La Transparencia y comparabilidad de la información En: Revista Legis del Contador Nº 9, pp. 145-167.

Mantilla Blanco, Samuel Alberto (2002). Contadores sí, ¿pero cuáles?, la globalización de la contaduría y sus consecuencias para el ejercicio profesional. En: Guía para la inserción contable Colombia en los escenarios internacionales. Bogotá D.C.: Pontifica Universidad Javeriana, pp. 139 -162.

Mantilla Blanco, Samuel Alberto (2001). Adoptar no es adaptar. A propósito de las diferencias entre los principios o normas colombianas y los estándares 
internacionales de contabilidad. En: Revista Legis del Contador, No 6, abril - junio, pp. $104-121$.

Mejía Soto, Eutimio (2004). Implicaciones de la Armonización o Estandarización de la Estructura Contable. Armenia. pp. 13-15.

Superintendencia de Sociedades (2006). Borrador Decreto. Por medio el cual deroga el Plan Único de Cuentas y dicta otras disposiciones en materia contable. 